\title{
A New SVD based Hybrid Color Image Watermarking for Copyright Protection using Contourlet Transform
}

\author{
C.Venkata Narasimhulu \\ Professor, Department of ECE \\ HIET, Hyderabad, India
}

\author{
K.Satya Prasad \\ Professor, Dept of ECE \\ JNTU Kakinada, India
}

\begin{abstract}
The authors propose a new hybrid watermarking scheme for copyright protection of color images using contourlet transform and singular value decomposition. The host color image and color watermark images are decomposed into directional sub- bands using contourlet transform and then applied Singular value decomposition to mid frequency subband coefficients. The singular values of mid frequency subband coefficients of color watermark image are embedded into singular values of mid frequency sub-band coefficients of host color image in Red, Green and Blue color spaces simultaneously based on spread spectrum technique. The experimental results shows that the proposed hybrid watermarking scheme is robust against common image processing operations such as, JPEG, JPEG 2000 compression, cropping, Rotation, histogram equalization, low pass filtering , median filtering, sharpening, shearing, salt \& Pepper noise, Gaussian noise, grayscale conversion etc. It has also been shown the variation of visual quality of watermarked image for different scaling factors. The comparative analysis reveals that the proposed watermarking scheme out performs the color image watermarking schemes reported recently.
\end{abstract}

\section{Keywords:}

Color image watermarking, Contourlet Transform, Singular value decomposition, Peak signal to noise ratio, normalized Correlation coefficient.

\section{INTRODUCTION}

With the rapid development of digital multimedia technique and the spread of internet, digital productions are easily copied and manipulated, so there exist a strong demand to protect the ownership and the copyright of this digital production. Digital watermarking is an excellent tool of copyright protection by embedding some information into the digital production. This embedding information can be later extracted from or detected in the multimedia to make an assertion about the data authenticity. Digital watermarks remain intact under transmission/transformation, allowing us to protect our ownership rights in digital form. Absence of watermark in a previously watermarked image would lead to the conclusion that the data content has been modified. A watermarking algorithm consists of watermark structure, an embedding algorithm and extraction or detection algorithm. In multimedia applications, embedded watermark should be invisible, robust and have a high capacity. Invisibility refers to degree of distortion introduced by the watermark and its affect on the viewers and listeners. Robustness is the resistance of an embedded watermark against intentional attack and normal signal processing operations such as noise, filtering, rotation, scaling, cropping and lossey compression etc. Capacity is the amount of data can be represented by embedded watermark.

\begin{abstract}
Watermarking techniques may be classified in different ways. The classification may be based on the type of watermark being used, i.e., the watermark may be a visually recognizable logo or sequence of random numbers. A second classification is based on whether the watermark is applied in the spatial domain or the transform domain. In spatial domain, the simplest method is based on embedding the watermark in the least significant bits (LSB) of image pixels. However, spatial domain techniques are not resistant enough to image compression and other image processing operations. Transform domain watermarking schemes such as those based on the discrete cosine transform (DCT), the discrete wavelet transform (DWT), contourlet transforms along with numerical transformations such as Singular value Decomposition (SVD) and Principle component analysis (PCA) typically provide higher image fidelity and are much robust to image manipulations [1].
\end{abstract}

Of the so far proposed algorithms, wavelet domain algorithms perform better than other transform domain algorithms since DWT has a number of advantages over other transforms including time frequency localization, multi resolution representation, superior HVS modeling, linear complexity and adaptivity and it has been proved that wavelets are good at representing point wise discontinuities in one dimensional signal. However, in higher dimensions, e.g. image, there exists line or curve-shaped discontinuities. Since, 2D wavelets are produced by tensor products of $1 \mathrm{D}$ wavelets they can only identify horizontal, vertical, diagonal discontinuities (edges) in images, ignoring smoothness along contours and curves. Curvelet transform was defined to represent two dimensional discontinuities more efficiently, with least square error in a fixed term approximation. Curvelet transform was proposed in continuous domain and its discretisation was a challenge when critical sampling is desired. Contourlet transform was then proposed as an improvement of curvelet transform. The contourlet is a directional multi resolution expansion, which can represent images containing contours efficiently [2]. Contourlet transform possess all features of wavelets and also shows a high degree of directionality and anisotropy. One of the unique properties of contourlet transform is that we could have any number of directional decompositions at every level of resolutions [3].

In this paper, proposed method is compared with another which is based on discrete wavelet transform (DWT), Biorthogonal wavelet transform. The peak signal to noise ratio (PSNR) between the original image and watermarked image and the normalized correlation coefficients (NCC) after different attacks were calculated. The results show high improvement detection reliability using proposed method. The rest of this paper is organized as follows. Section 2 describes the contourlet transform, section 3 describes singular value decomposition, section 4 illustrates the details of proposed method, in section 5 experimental results are discussed 
without and with attacks, conclusion and future scope are given in section 6 .

\section{CONTOURLET TRANSFORM}

Contourlet transform is a multi resolution and multidirectional transformation technique which is used in image analysis for capturing contours and fine details in images [2].The contourlet transform is composed of basis functions oriented at different directions in multiple scales with flexible aspect ratios. This frame work should form a basis with small redundancy unlike other transform techniques in image processing, Contourlet representation contains basis elements oriented at variety of directions much more than few directions that are offered by other separable transform technique. One way to obtain a sparse expansion for images

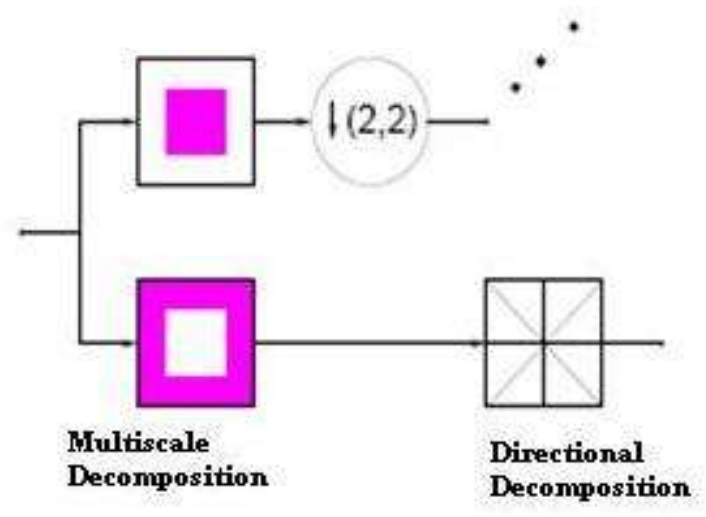

Fig1.Block diagram of Contourlet Filter Bank

The directional decomposition is implemented through an 1level tree structured decomposition that leads to $2^{1}$ sub bands with wedge shaped frequency partition.

Fig.3 shows directional decomposition at every level obtained using contourlet transform. The no. of directional decompositions can be chosen different and it makes this transform unique. From fig. 2, it is apparent that $\mathrm{L}$ with smooth contours is first apply a multistage wavelet like transform to capture the edge points, and then local directional transform to gather the nearby edge points into contour segments. With this insight, one can construct a double filter bank structure shown in figure 1 where the Laplacian pyramidal (LP) filter is used to capture the point discontinuities, followed by a directional filter bank (DFB) to link point discontinuities into linear structures. The overall result is an image expansion using basic elements like contour segments, and thus it is named contourlet transform. The combination of this double filter bank is named pyramidal directional filter bank (PDFB). An example of frequency partitioning of a PDFB is shown in fig2 [3].

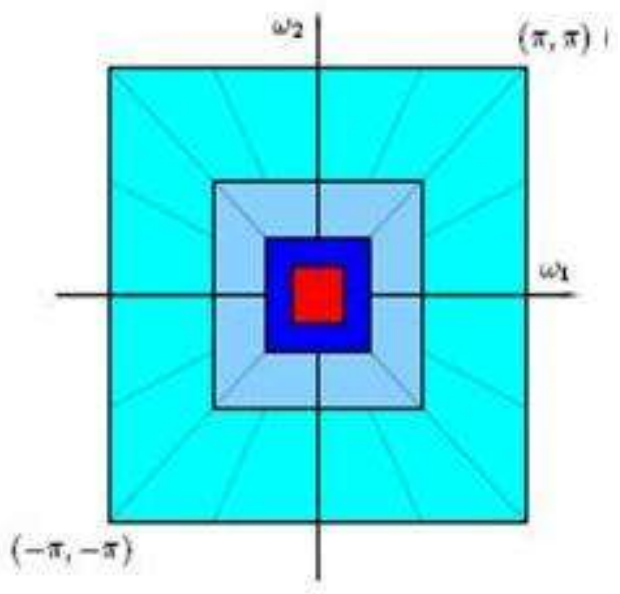

Fig2. Resulting frequency division where the no. of Directions is increases with frequency

is the mid pass version of the images and $\mathrm{V}, \mathrm{X}, \mathrm{Y}$ and $\mathrm{Z}$ are the directional detail bands at different levels. Directional bands in four levels of multi resolutions are divided into 2, 4, 8 and 16 directional sub bands from coarse to fine scales respectively. In this method, mid frequency version sub-band ' $\mathrm{X}$ ' is selected for watermark embedding as discussed in section 4 [4]. 


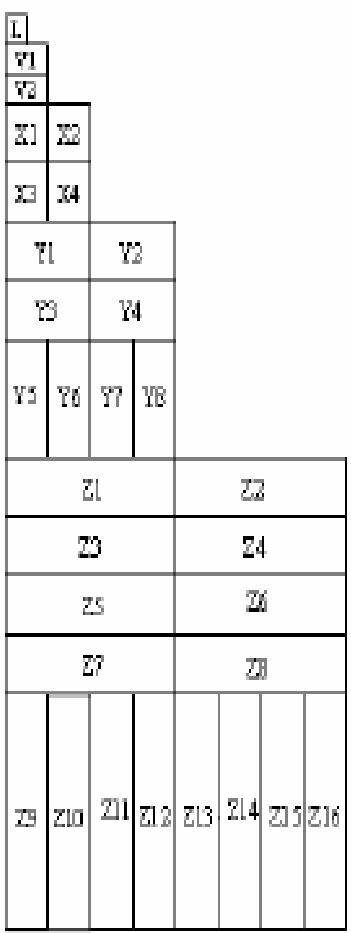

Fig 3:Contourlet transform decomposition

\section{SINGULAR DECOMPOSITION}

Singular value decomposition (SVD) is a popular technique in linear algebra and it has applications in matrix inversion, obtaining mid dimensional representation for high dimensional data, for data compression and data denoising. If $\mathrm{A}$ is any $\mathrm{N} \times \mathrm{N}$ matrix, it is possible to find a decomposition of the form

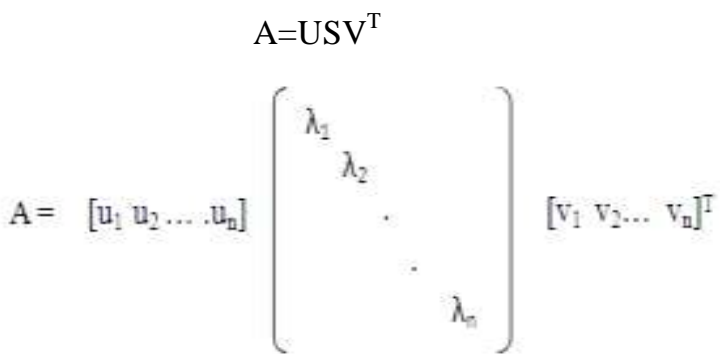

Where $\mathrm{U}$ and $\mathrm{V}$ are orthogonal matrices of order $\mathrm{N} \mathrm{x} \mathrm{N}$ and $\mathrm{N}$ $x$ such that $U^{\mathrm{T}} \mathrm{U}=\mathrm{I}, \mathrm{V}^{\mathrm{T}} \mathrm{V}=\mathrm{I}$, and the diagonal matrix $\mathrm{S}$ of order $\mathrm{N}$ x $\mathrm{N}$ has elements $\lambda_{\mathrm{i}}(\mathrm{i}=1,2,3, . . \mathrm{n})$, I is an identity matrix of order $\mathrm{N} x \mathrm{~N}$.

The diagonal entries are called singular values of matrix A, the columns of $U$ matrix are called the left singular values of $\mathrm{A}$, and the columns of $\mathrm{V}$ are called as the right singular values of A

The general properties of SVD are [5],[6] a) Transpose: $A$ and its transpose $A^{T}$ have the same nonzero singular values.

b) Flip: A, row-flipped Arf, and column-flipped Acf have the same non-zero singular values.

c) Rotation: $A$ and $\operatorname{Ar}$ ( $A$ rotated by an arbitrary degree) have the same non-zero singular values.

d) Scaling: $B$ is a row-scaled version of $A$ by repeating every row for $L 1$ times. For each non-zero singular value $\lambda$ of $A, B$ has $\sqrt{ } L 1 \lambda . C$ is a column-scaled version of $A$ by repeating every column for $L 2$ times. For each nonzero singular value $\lambda$ of $A, C$ has $\sqrt{ } L 2 \lambda$. If $D$ is row-scaled by $L 1$ times and columnscaled by $L 2$ times, for each non-zero singular value $\lambda$ of $A, D$ has $\sqrt{ } L 1 L 2 \lambda$

e) Translation: $A$ is expanded by adding rows and columns of black pixels. The resulting matrix $A e$ has the same Nonzero singular values as A.

The important properties of SVD from the view point of image processing applications are:

1. The singular values of an image have very good stability i.e. When a small perturbation is added to an image, their singular values do not change significantly.

2. Singular value represents intrinsic algebraic image properties.[5],[6]

Due to these properties of SVD, in the last few years several watermarking algorithms have been proposed based on this technique. The main idea of this approach is to find the SVD of a original image and then modify its singular values to embedded the watermark. Some SVD based algorithms are purely SVD based in a sense that only SVD domain is used to embed watermark into original image. Recently some hybrid SVD based algorithms have been proposed where different types of transform domains including discrete cosine transform (DCT), discrete wavelet transform (DWT), etc are used to embed watermark into original color image. Here the proposed scheme uses Contourlet transform (CT) along with SVD for watermarking to obtain better performance compared to existing hybrid algorithms.

\section{PROPOSED ALGORITHM}

In this paper, Contourlet Transform and SVD based hybrid technique is proposed for color image watermarking that uses true color images for both watermark and host images. The robustness and visual quality of watermarked image is tested with three quantifiers such as PSNR, NCC and Bit Error Rate. It is investigated whether the CT-SVD advantages over color watermarking using wavelet transform with their extra features would provide any significance in terms of watermark robustness and invisibility.4.1, 4.2 explain the watermark embedding and extraction algorithm $[7],[8],[9],[10],[11]$.

\subsection{Watermark Embedding Algorithm}

The proposed watermark embedding algorithm is shown in Figure 4 . The steps of watermark embedding algorithm are as follows. 


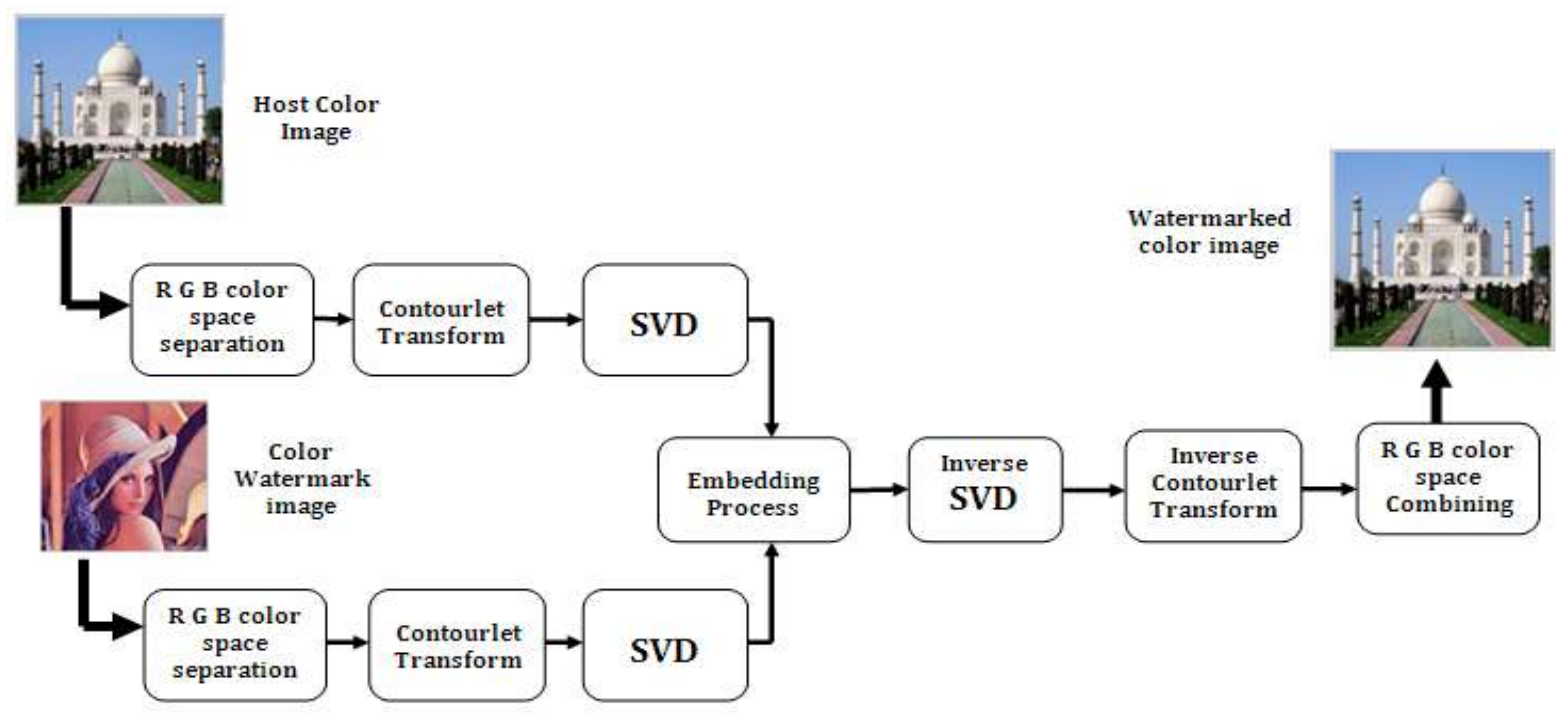

Fig4Watermark Embedding Algorithm

Step1: Separate the R G B color spaces of both host and watermark color images.

Step2: Apply Contourlet Transform to the R color space of both host image and watermark image to decompose them into sub bands.

Step3: Apply SVD to mid frequency sub-band of CT of R color space of both host and watermark image.

Step4: Modify the singular values of mid frequency sub-band coefficients of R color space of host image with the singular values of mid frequency sub-band coefficients of $\mathrm{R}$ color space of watermark image using spread spectrum technique..

$$
\text { i.e. } \quad \lambda_{I},=\lambda_{I}+\alpha \lambda_{W} \text {. }
$$

Where $\alpha$ is scaling factor,[4] $\lambda_{I}$ is singular value of $R$ color space of host image, $\lambda_{\mathrm{W}}$ is singular value of $\mathrm{R}$ color

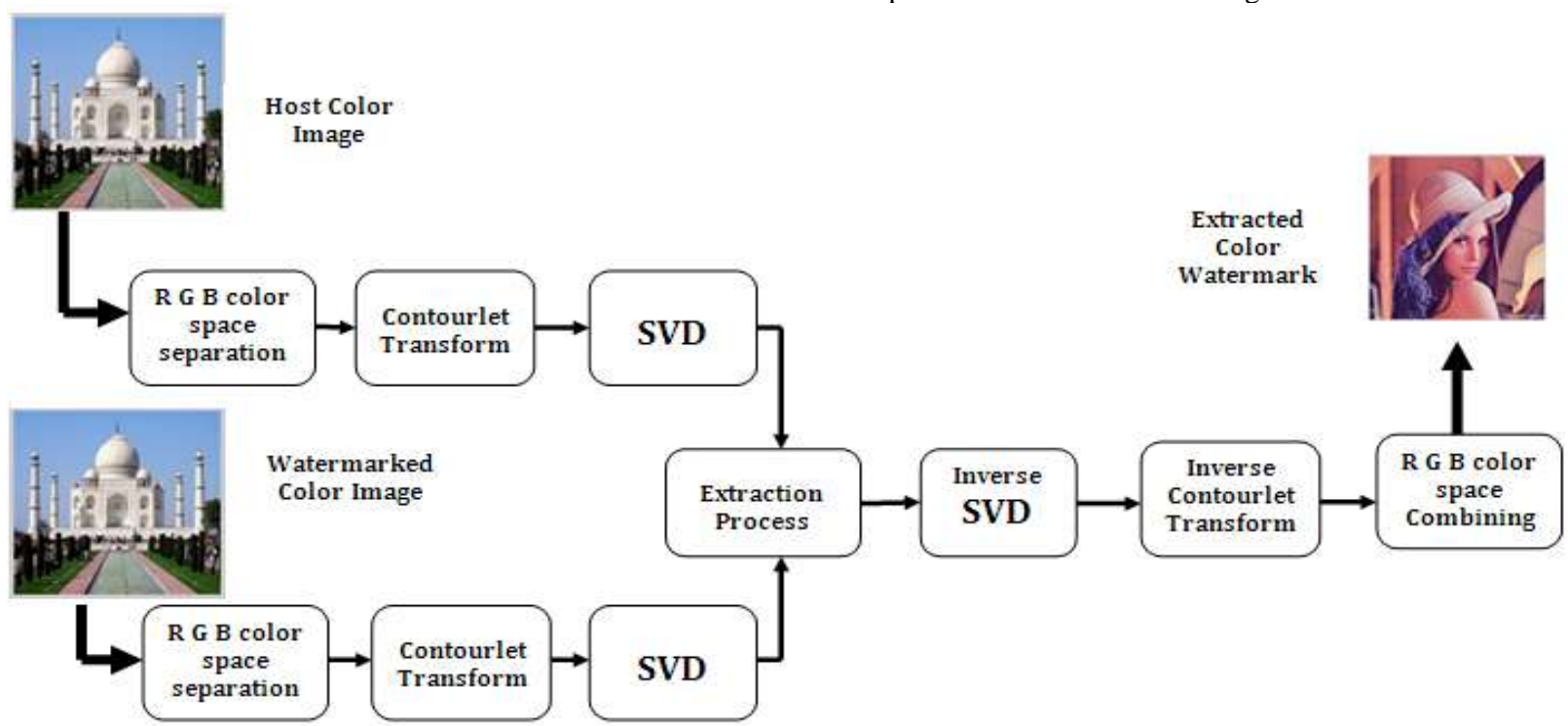

Fig 5: Watermark Extraction Algorithm

Step1: Separate the R,G,B color spaces of watermarked image.

Step2: Apply Contourlet Transform to the $\mathrm{R}$ color space obtained in step1.

Step3: Apply SVD to mid frequency sub-band of R color space of transformed watermarked image. space of watermark and $\lambda_{\mathrm{I}}$, becomes singular value of $\mathrm{R}$ color space watermarked image.

Step5: Apply inverse SVD on modified singular values obtained in step4 to get the mid frequency sub-band

Step6: Apply inverse Contourlet Transform to the mid frequency sub-band coefficients obtained in step 5 to get the $\mathrm{R}$ color space of watermarked image.

Step7: Apply the same Steps from Step2 to Step6 for the G and B color subspaces.

Step8: Combine the R, G and B color spaces of watermarked image to obtain the color watermarked image.

\subsection{Watermark Extraction Algorithm}

The watermark extraction algorithm is shown in Figure 5 The Steps of watermark extraction algorithm are as follows. coefficients of watermarked image. 
Step5: Apply inverse SVD to obtain mid frequency coefficients of $\mathrm{R}$ color space of transformed watermark image using Step 3.

Step6: Apply inverse CT using the coefficients of the mid frequency sub-band to obtain the R color space of Watermark image.

Step7: Repeat the Steps 2 to 6 for $\mathrm{G}$ and B color spaces.

Step8: Combine the R,G and B color spaces to get the color watermark.

\section{EXPERIMENTAL RESULTS}

In the experiments, we use the true color "tajmahal.jpg" of size 256X256 as host image as shown in the Figure 6 and true color "lena.jpg" of size $128 \times 128$ as watermark as shown in Figure 7. The experiment is performed by taking scaling factor alpha as 0.5.The results show that there are no perceptibly visual degradations on the watermarked image shown in Figure 8 with a PSNR of $46.7055 \mathrm{~dB}$. Extracted watermark without attack is shown in Figure 9 with NCC around unity. The proposed method is also tested for binary and grayscale watermark image of size $128 \times 128$ and watermarked and extracted watermark are shown in table 1.

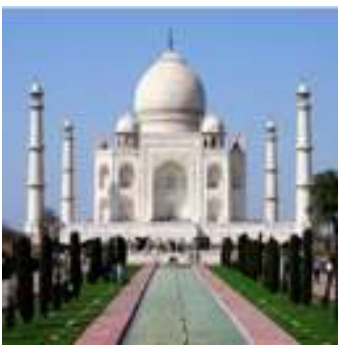

Fig (6) Host image "tajmahal.jpg"

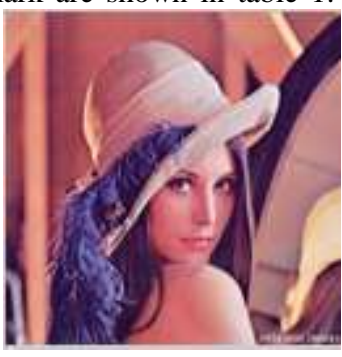

Fig(7) Watermark image "LENA.jpg"

MATLAB 7.6 version is used for testing the robustness of the proposed method. As the scaling factor increases the brightness increases and hence visual quality degrades. The variation of visual quality of watermarked images for different scaling factors is illustrated in table 2 .

The proposed algorithm is tested for different host images such as "lotus.jpg", "Baboon.jpg", "Barbara.jpg", "Charminar.jpg" and "Parrot.jpg" as shown in Table 3 and it is observed that there are no visual degradations on the respected watermarked images. For all the different host test images, the watermark is effectively extracted with around unity NCC. Various intentional and non-intentional attacks are tested for robustness of the proposed watermark algorithm includes JPEG, JPEG2000 compressions, low pass filtering, Rotation, Histogram Equalization ,Median Filtering, Salt \&Pepper Noise, Weiner Filtering, Gamma Correction, Gaussian Noise, Rescaling ,Sharpening ,Blurring ,Contrast Adjustment ,Automatic cropping, Dilation, Bit Plane Removal, Row Colum Copying, Row Colum removing, color to Gray scale conversion ,shearing and sharpening.

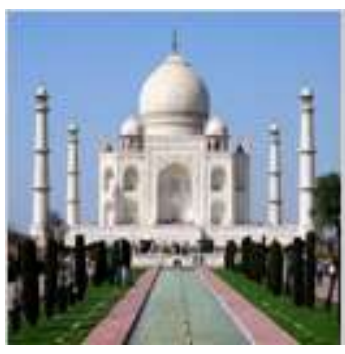

Fig(8) Watermarked image PSNR $=46.7055$

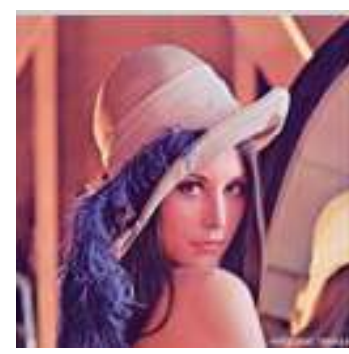

Fig(9) Extracted watermark $\mathrm{NCC}=\mathbf{0 . 9 9 9 6}$

The proposed algorithm is compared with P.Ramana Reddy's and K.Ramani's papers [12],[13] in which the watermarking is done by using DWT and Bi-Orthogonal wavelet transform respectively and the PSNR is reported as 46.8500 and 31.1750 and the No of attacks tested are only 2 and 7 for watermark

Table 1: Watermarked and Extracted watermark images for binary and grayscale watermark

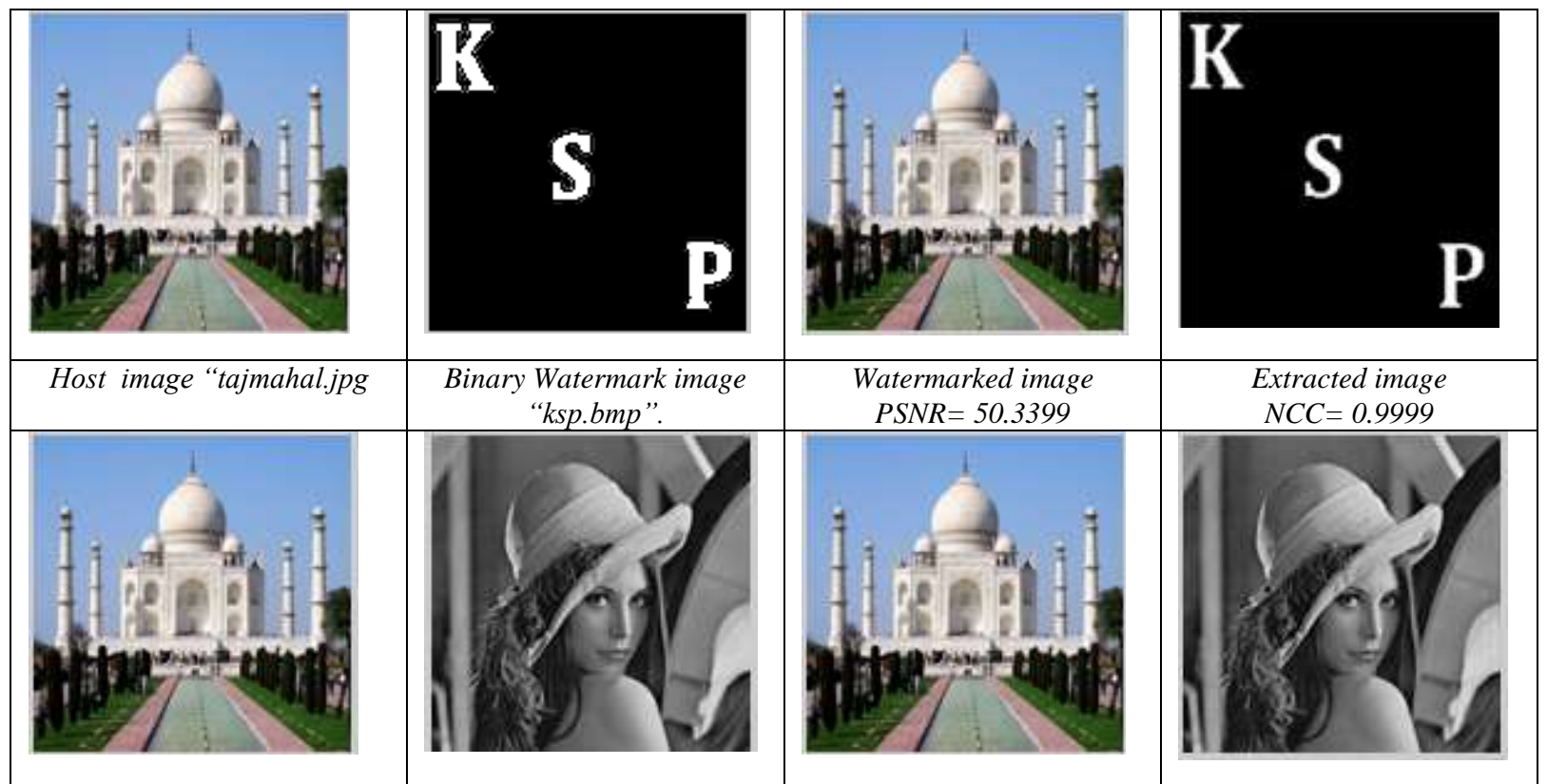




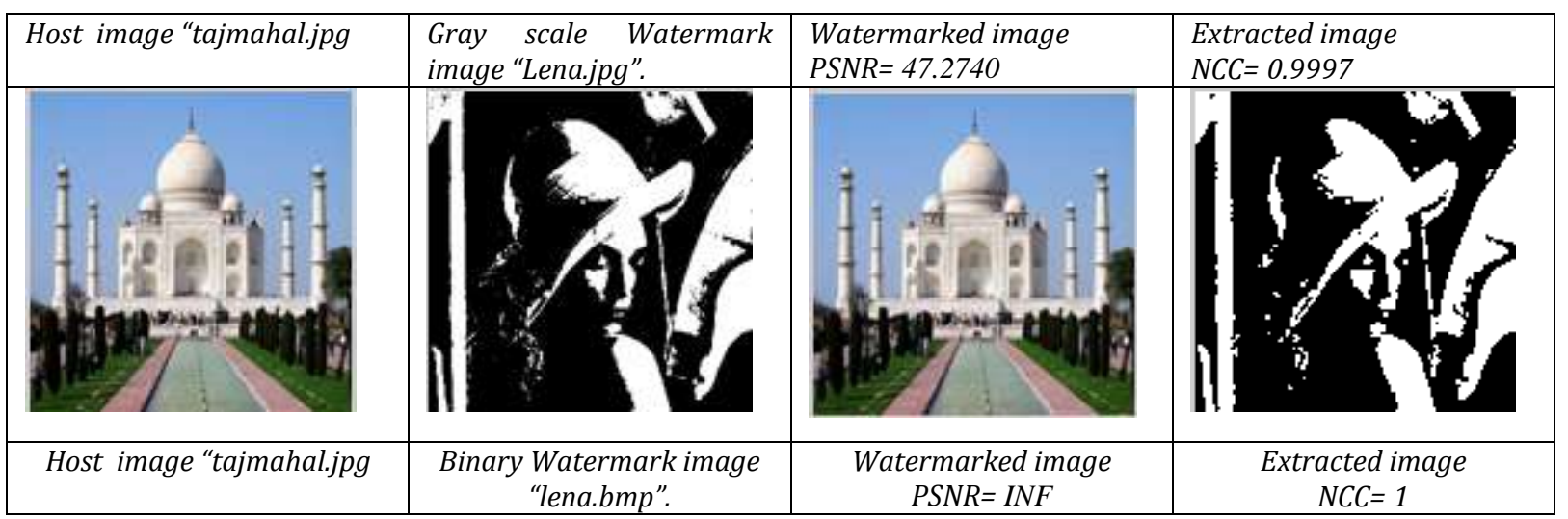

Table 2: Watermarked image for different scaling factor (Alpha)

\begin{tabular}{|c|c|c|}
\hline Alpha=0.5,PSNR=46.7055 & Alpha=1.0,PSNR=40.8760 & Alpha=7.00,PSNR= 29.3363 \\
\hline Alpha=10.00, PSNR=28.7037 & Alpha=25, PSNR=27.7302 & Alpha $=50.00, \mathrm{PSNR}=27.3313$ \\
\hline & & \\
\hline
\end{tabular}

Table 3: Watermarked and Extracted Watermark with PSNR, NCC for different Original Images.

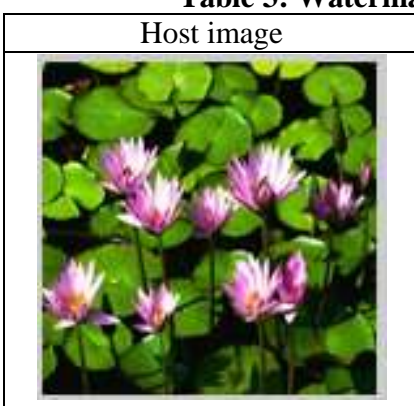

"lotus.jpg"

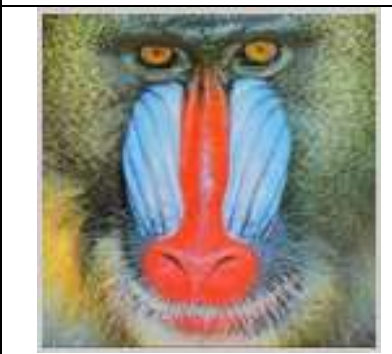

"baboon.jpg"

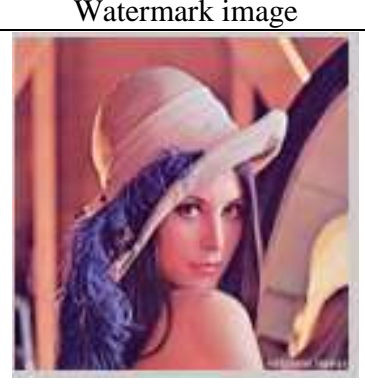

"LENA.jpg"

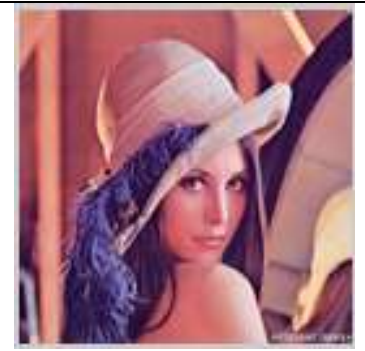

"LENA.jpg"

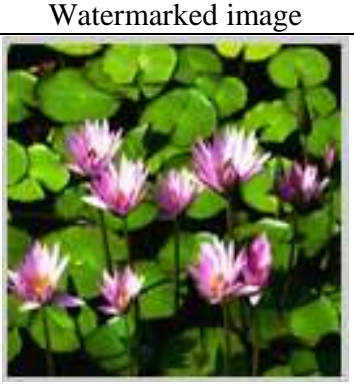

PSNR $=46.9753$

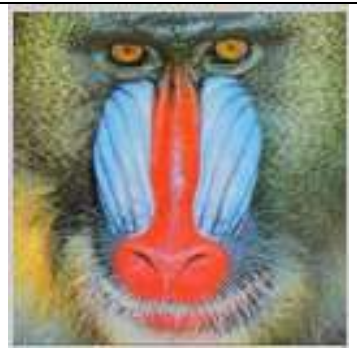

PSNR $=45.9880$

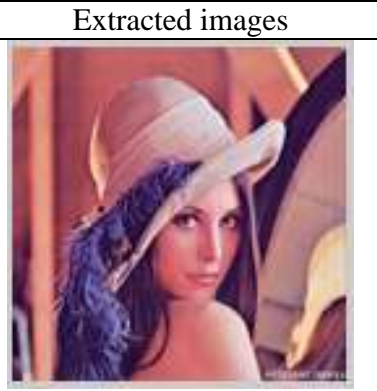

$\mathrm{NCC}=0.9996$,

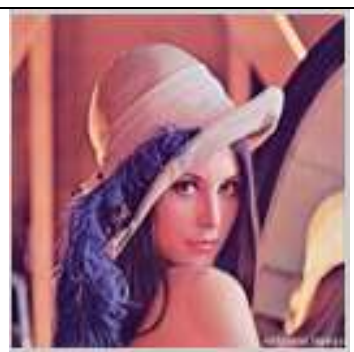

$\mathrm{NCC}=0.9996$ 


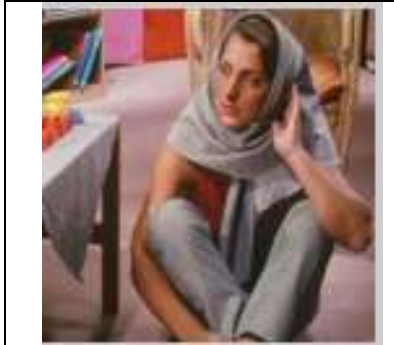

"barbara.jpg"

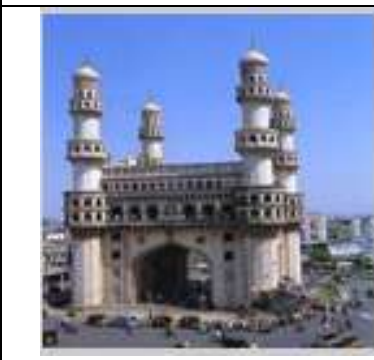

“charminar.jpg"

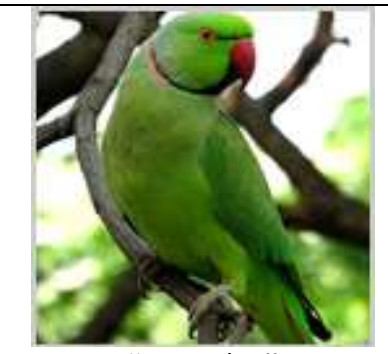

"Parrot.jpg"

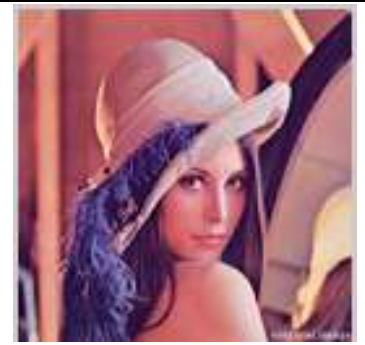

"LENA.jpg"

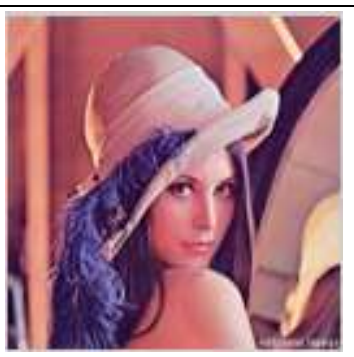

"LENA.jpg"

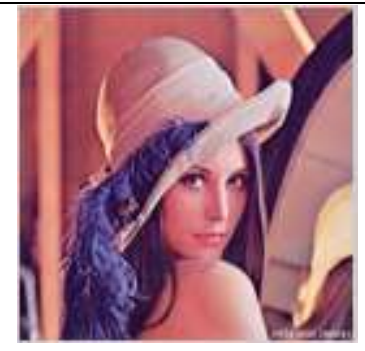

"LENA.jpg"

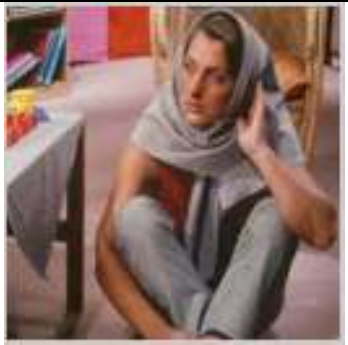

$\mathrm{PSNR}=46.1780$

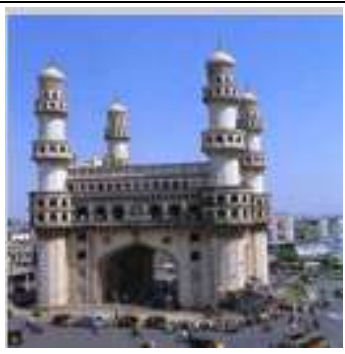

$\mathrm{PSNR}=46.4878$

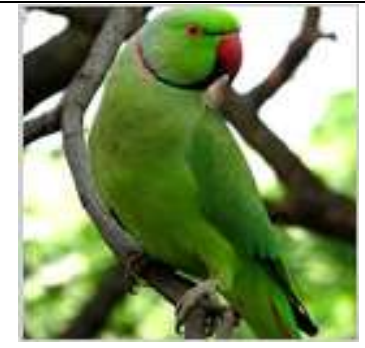

$\mathrm{PSNR}=47.9006$

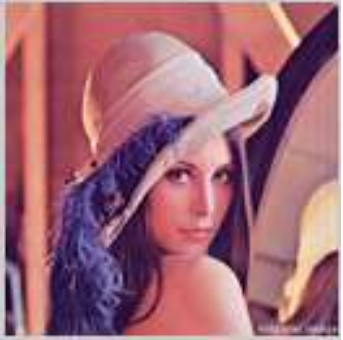

$\mathrm{NCC}=0.9996$

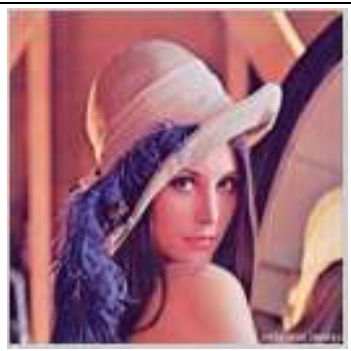

$\mathrm{NCC}=0.9996$
In Table 4, the normalized correlation coefficient values for different attacks are shown with extracted watermark and attacked watermarked image. The quality and imperceptibility of watermarked image is measured by using PSNR. The PSNR is calculated separately for R, G, B space using eq.3 [14] with respect to the respective color space of host image. The final PSNR of watermarked image is taken as mean of PSNR obtained with three color spaces. The similarity of extracted watermark with original watermark embedded is measured using NCC. The NCC is calculated using eq. (4) for the three color spaces [15] and their mean is taken as the resultant Normalized Correlation coefficient.

$$
P S N R=10 \log \left[\frac{\max (I(i, j))^{2}}{\sum\left(I^{\prime}(i, j)-I(i, j)\right) 2}\right] .
$$

Normalized Correlation Coefficient

$$
\mathrm{Ncc}=\frac{\sum_{m} \sum_{n}\left(A_{m n}-\bar{A}\right)\left(B_{m n}-\bar{B}\right)}{\sqrt{\left(\sum_{m} \sum_{n}\left(A_{m n}-\bar{A}\right)^{2}\right)\left(\sum_{m} \sum_{n}\left(B_{m n}-\bar{B}\right)^{2}\right)}}
$$

Table 4: Extracted watermarks with NCC for different attacks along with attacked watermarked image

\begin{tabular}{|l|l|l|l|}
\hline \multicolumn{2}{|c|}{ Jpeg compression Ncc=0.9996 Jpeg2000Compression Ncc=0.9996 } \\
\hline
\end{tabular}




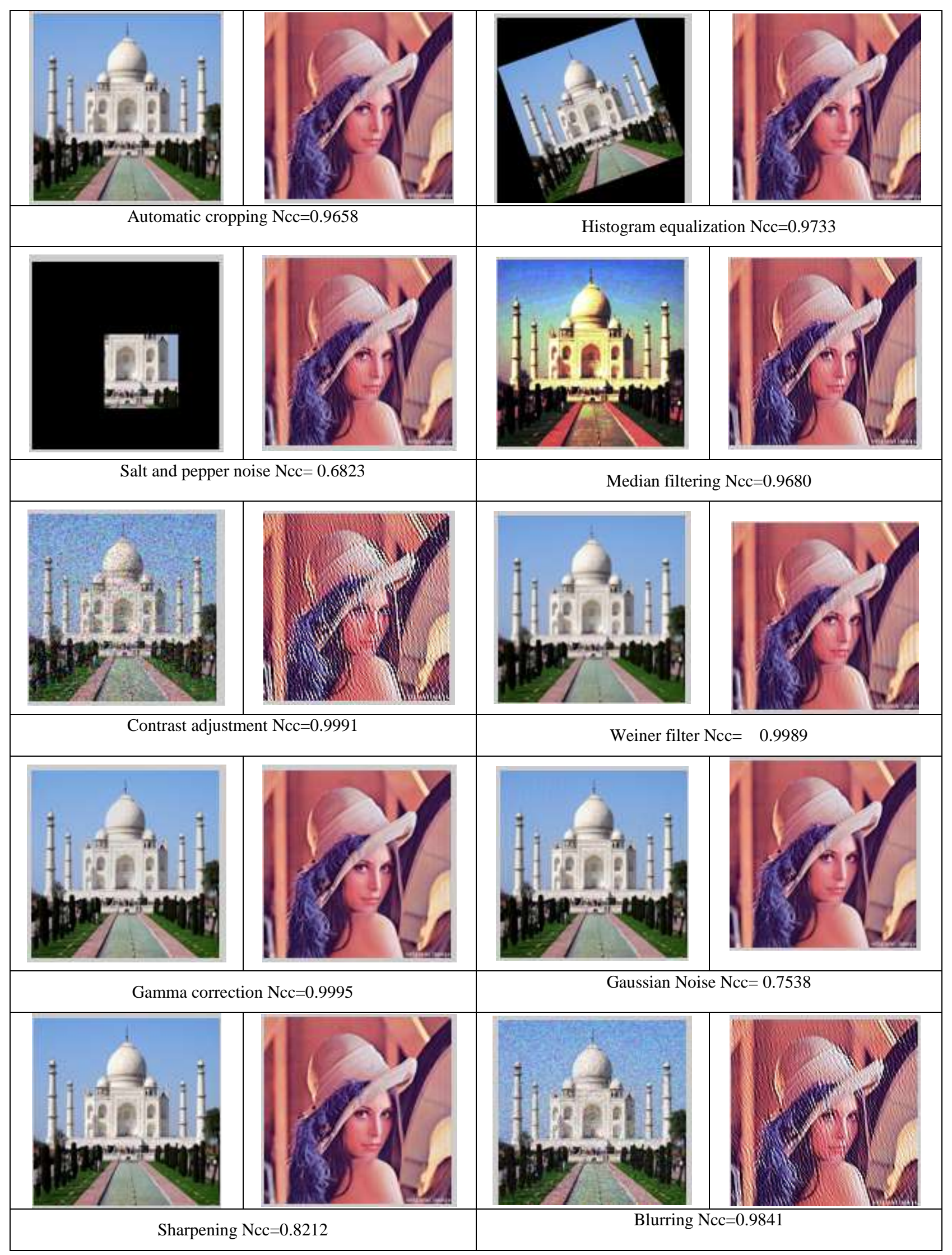




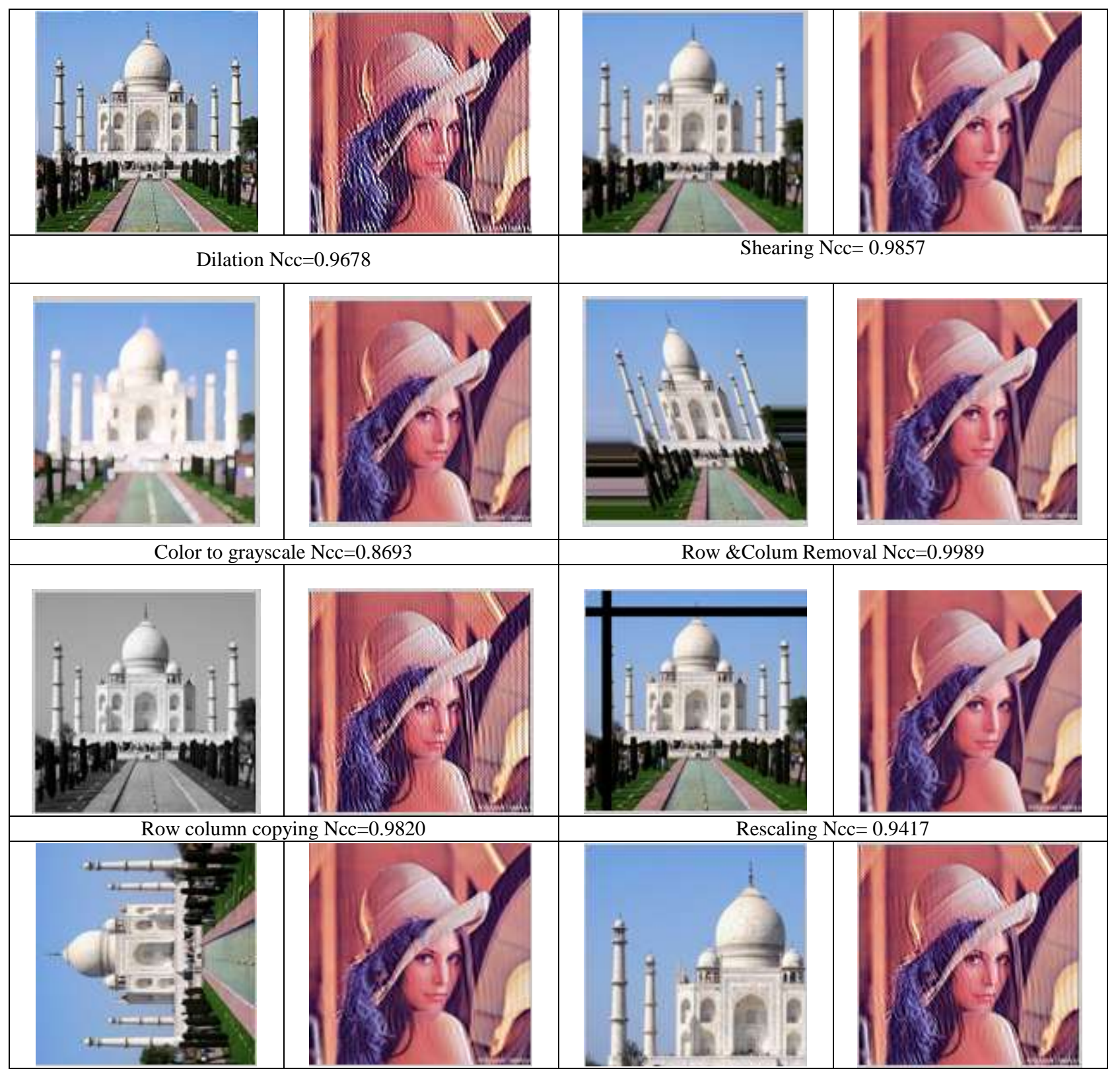

Table 5 Comparison of proposed method with P.Ramanareddy and K.Ramani's

\begin{tabular}{|l|l|l|l|}
\hline Characteristic & Proposed method & $\begin{array}{l}\text { P.Ramanareddy \& } \\
\text { D.Sreenivas Rao }\end{array}$ & $\begin{array}{l}\text { K.Ramani \& } \\
\text { E.V.Prasad }\end{array}$ \\
\hline Size of host image & $256 \times 256$ & $256 \times 256$ & $256 \times 256$ \\
\hline Size of watermark & $128 \times 128$ & $64 \times 64$ & $60 \times 60$ \\
\hline Type of watermark & Binary, grayscale and Color & Binary & Gray scale \\
\hline Transform used & $\begin{array}{l}\text { Contourlet Transform and } \\
\text { SVD }\end{array}$ & $\begin{array}{l}\text { Discrete Wavelet } \\
\text { Transform }\end{array}$ & $\begin{array}{l}\text { Biorthogonal wavelet } \\
\text { transform }\end{array}$ \\
\hline Location of embedding & $\begin{array}{l}\text { Mid frequency in all color } \\
\text { spaces }\end{array}$ & $\begin{array}{l}\text { Mid frequency in only } \\
\text { blue channel }\end{array}$ & $\begin{array}{l}\text { Mid frequency in only blue } \\
\text { channel }\end{array}$ \\
\hline PSNR in DB & $\begin{array}{l}50.3399 \text { (binary watermark), } \\
47.2740 \text { (grayscale watermark) } \\
46.7055 \text { (color watermark) }\end{array}$ & 46.8500 & 31.1750 \\
\hline NCC without attack & 0.9996 & Not given & 0.8585 \\
\hline No of attacks tested & 20 & 2 & 7 \\
\hline
\end{tabular}




\section{CONCLUSION}

In this paper, a new SVD based hybrid watermarking scheme is proposed for copyright protection of color images using contourlet transform. Watermark is embedded in all color spaces of host image by modifying singular values of mid frequency sub band coefficients with respect to watermark mid frequency sub band coefficient with suitable scaling factor. The robustness of watermark is improved for common image procession operations by combining both the concepts of contourlet transform and singular value decomposition. The proposed method tested for binary, grayscale and color watermark images. It also demonstrates that the visual quality of water marked image degrades as scaling factor increases. The proposed algorithm is tested for different host images and respective watermark images are obtained without any visual degradation. The proposed algorithm preserves high perceptual quality of the watermarked image and shows an excellent robustness to attacks like JPEG, JPEG2000 compressions, Low pass filtering, Histogram equalization, Gray scale conversion, Salt and Pepper Noise, Shearing, Gamma correction, Cropping, Dilation, Weiner Filtering, Gaussian Noise, Rescaling, Row Column Copying, Row Column Removal and Contrast adjustment. The proposed method shows higher robustness to maximum no of attacks compared to P.Ramanareddy's and K.Ramani's algorithm.

\section{REFERENCES}

[1] C.Venkata Narasimhulu and k.Satya Prasad, "A Novel Robust Watermarking Technique Based on Nonsubsampled Contourlet Transform and SVD", International journal of Multimedia and its Applications Vol 3, No 1, Feb 2011.

[2] Minh N. Do, and Martin Vetterli, "The Contourlet Transform: An Efficient Directional Multiresolution Image Representation" IEEE transaction on image processing, Vol 14, issue no 12, pp 2091-2106, Dec 2005.

[3] Elham salahi, M.Shahram Moin and Ahmad salahi "A new Visually Imperceptible and Robust Image Water marking Scheme in contourlet Domain" International conference on intelligent information hiding and multimedia signal Processing, 2008.

[4] M Jayalakshmi, S. N. Merchant, Uday B. Desai, "Digital Watermarking in Contourlet Domain," ICPR, vol. 3. Pp.861-864, 18th International Conference on Pattern Recognition (ICPR'06) Volume 3, 2006.

[5] Emir Ganic and ahmet M. Eskicioglu "Robust embedding of visual watermarks using discrete wavelet transform and singular value decomposition Journal. Of Electron. Imaging, Vol. 043004 (2005);doi:10.1117/1.2137650 Published 12 December 2005 .

[6] R. Liu and T. Tan, "An SVD based watermarking Scheme for protecting rightful ownership", IEEE Trans, Multimedia.Vol. 4, no.1, pp.121-128, Mar.2002.

[7] Das, S. Bandyopadhyay, P. Paul, S.Ray, A.S. Banerjee, M. "A New Introduction towards Invisible Image Watermarking on Color Image" IEEE international advance computing Conferences (IACC2009) 6-7 MARCH 2009.
[8] C.Venkata Narasimhulu and K.Satya Prasad, "A hybrid watermarking scheme using contourlet Transform and Singular value decomposition" IJCSNS: International Journal of Computer Science and Network Security, Vol.10, No.9, September 2010.

[9] Vikas Saxena,J.P.Gupta,"collusion attack resistant watermarking scheme for colored images using DCT" International journal of computer sciences 34:2,IJCS $34 \_2$ - 22 .

[10] keshava S Rawat,Dheerendra s tomar"digital watermarking schemes for authorization against copying or piracy of color images" international journal of computer sciences and engineering vol 1 No 42008.

[11] M.Hamad Hassan, S.A.M.gilani”A semi-fragile watermarking scheme for color image authentication "world academy of Science, Engineering and technology 192006.

[12] P.Ramanareddy, M.V.N.K Prasad,D.Sreenivasarao "Robust digital watermarking of color images Under noise attacks "international journal of Recent trends in engineering volume 1, No 1, May 2009.

[13] K.Ramani,E.V.Prasad,V.Lokanadham naidu, D. Ganesh "color image watermarking using bi-orthogonal wavelet transform" international journal of computer applications volume 11 No 9 Dec 2010.

[14] Ashraf. K. Helmy and GH.S.El-Taweel "Authentication Scheme Based on Principal Component Analysis for Satellite Images" International Journal of Signal Processing, Image Processing and Pattern Recognition Vol. 2, No.3, September 2009

[15] Matlab 7.6 version, Image Processing Tool Box.

\section{AUTHORS PROFILE}

C.V. Narasimhulu received his Bachelor degree in Electronics and Communication Engineering from S.V. University, Tirupati, India in 1995 and Master of technology in Instruments and Control Systems from Regional Engineering College Calicut, India in 2000.He is currently pursuing the Ph.D degree in the department of Electronics and Communication Engineering from Jawaharlal Nehru Technological University Kakinada, India. He has more than 15 years experience of teaching under graduate and post graduate level. He is interested in the areas of signal processing and multimedia security.

K. Satya Prasad received his Ph.D degree from IIT Madras, India, $\mathrm{He}$ is presently working as professor in the department of Electronics and Communication Engineering, JNTU college of Engineering Kakinada and Rector of Jawaharlal Nehru Technological University, Kakinada, India. He has more than 30 years of teaching and research experience. He published 30 research papers in international and 20 research papers in National journals. He guided $8 \mathrm{Ph} . \mathrm{D}$ theses and $20 \mathrm{Ph} . \mathrm{D}$ theses are under his guidance. His area of interests includes Digital Signal and Image Processing, Communications, Ad-hoc networks etc. 